\title{
Multi-layered sensing approach for environment perception with one multi-functional sensor
}

\author{
Non-member, Jinwei Sun and Member, Katsunori SHIDA \\ Faculty of Science and Engineering, Saga University, 1 Honjo-machi, Saga, 840-8502 Japan
}

\begin{abstract}
This paper presents a novel environment perception approach based on multi-layer sensing technique using one multifunctional sensor to measure temperature, humidity and brightness. It focuses on the processing of the multifunctional information in a multi-layer framework, which is more attractive in terms of both system simplicity and performance, and is proved to be effective to solve the problem of more than three quantities being reconstructed. A CdS and $\mathrm{Fe}_{3} \mathrm{O}_{4}$ material based multi-function sensor has been developed and the multi-layer data fusion idea has also been proposed for the use.
\end{abstract}

Keywords: multi-functional sensor, multi-layer sensing, measurement equations

\section{Introduction}

As a common sense, different sensors are used when there are different variables to be sensed ${ }^{[1],\{2]}$. Different from the popular technique, known as the compound sensing, multi-functional sensors have been developed in the last decade in case the quantities being measured affect more than one single sensor's input or compact structure of the sensing component is forcibly requested. The schematic structure of multi-functional sensor is shown in Fig.1, $x_{1}$ and $x_{2}$ are two quantities under test, with the sensor outputs

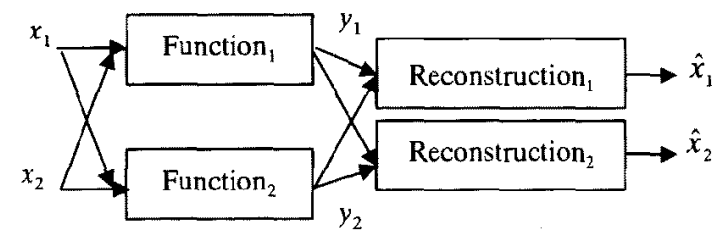

Fig. 1 A multifunctional sensor with two quantities

$\hat{x}_{1}$ and $\hat{x}_{2}$ being their estimations. We have acquired considerable achievements in this field. For example, with a metal spirals based sensor, many kinds of metals can be distinguished from the analysis of the measurement results as impedance, capacitance and inductance ${ }^{[3]}$; and also by adding piezo-resistance rubber device, more non-metal materials will be recognized further ${ }^{[4]}$. Moreover, the concentration of electrolyte had been nrecisely demarcated by using a non-destructive multi-functional sensor ${ }^{[s]}$.

Multifunctional sensing strategy consists in the algorithm to solve the measurement equations that result from the inputs and outputs of the multi-functions, on which quantities are mixed to exert their effects. The functions of the sensor are usually nonlinear ones, they are

$$
\begin{aligned}
& y_{1}=f\left(x_{1}, x_{2}\right) \\
& y_{2}=g\left(x_{1}, x_{2}\right)
\end{aligned}
$$

Where, $f()$ and $g()$ are general nonlinear functions.

Almost all the multi-functional sensors that had been developed examine no more than two variables, because only in this case could the least mean square method (LMS) or database technique be possibly applied to the reconstruction of measurand (quantities being measured). For more than three variables, the number of measurement equations may go up to three, as a consequence, the solution algorithm must be performed in $4 \mathrm{D}$ space, in which arbitrary choice may more frequently be imposed, and leads to the estimation ceasing to be effective.

Four methods for measurand reconstruction have been introduced by Tomasz Szafranshi ${ }^{[6]}$, but those techniques were practical only for single input/output pair. In the case of multi-functional converse problems, the identical study called "measurement equations" had been mathematically approached by Prof. Kobayashi ${ }^{[7]}$ early in $80 \mathrm{~s}$, and the solution to the nonlinear situation that accounts for a special case $\left(y=f\left(x_{1}, x_{2}, \ldots, x_{N}\right)=x_{1} f_{1}\left(x_{2}, \ldots, x_{N}\right)\right)$ was briefly discussed. However, algorithms suitable for general nonlinear functions have by far never appeared. Some researchers are trying to employ ANN (Artificial Neural Network) to map the nonlinear functions of sensors, but training problems certainly existed while serving for recognition of complex objects ${ }^{[8]}$.

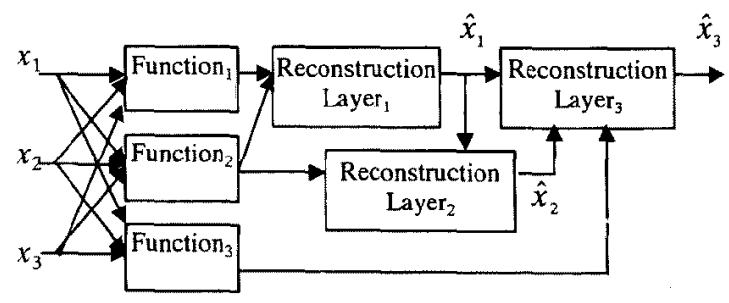

Fig.2 Multi-layer sensing to measure three quantities

In this paper, a novel multi-functional sensor is developed for measuring three environment quantities: humidity, temperature and brightness. The measurand reconstruction problem of the proposed sensor is solved by a new approach called multi-layer sensing, which acquires highlevel information in a multi-layered architecture. The physical quantities are evaluated one by one according to the requirements, thus decoupling the reconstruction of 3variables into 2 -variables or 1-variable subjects, as shown 
in Fig.2. This method is valid under the circumstance that two of the variables (e.g. $x_{1}$ and $x_{2}$ ) could be considered in the measurement equations but with others (e.g, $x_{3}$ ) available for being ignorable. The proposed approach itself has not satisfied the universal cases yet. However, the technique idea is effective not only for the proposed sensor, but also for many other measurement occasions.

The similar idea named hierarchical understanding has been broadly applied in pattern recognition field ${ }^{[9]}$. A simple example is shown in Fig. 3 , which is required to classify the figures into several groups. Different factors (layers) must be detected for the classification, which may be area, linearity and confinement. Fig. 4 denotes the flow chart of one possible classification strategy.

Another resemble research in sensing field has been approached as adaptive measuring system ${ }^{[10], \mid 11]}$. For example, a study on "Zooming Functional Measurement" was proposed to measure the two-dimension position more and more precisely by adjustment of the system parameters ${ }^{[10]}$. Their main interests lie in controlling the system in its optimum condition to make the measurement of one physical variable more reasonable (e.g. precise), other than process multi-variables for high-level information as we do. However, the common strategy is to get the final solution gradually.

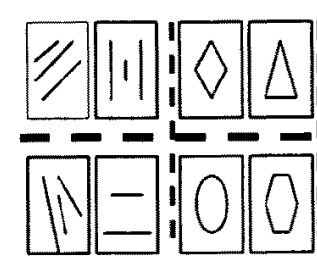

A A

Fig.3 Figures under classification.

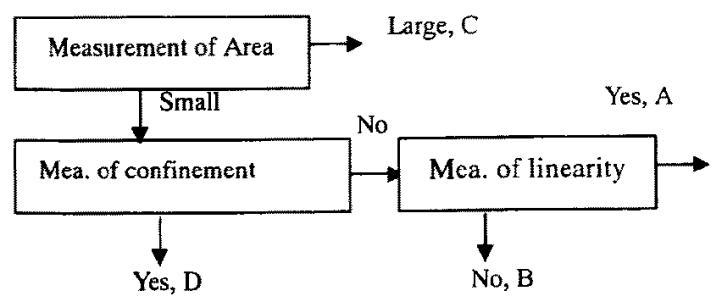

Fig. 4 Flowing chart of the classification procedure.

Besides avoiding the calculation of reconstruction in $4 \mathrm{D}$ spaces, multi-layer sensing may possess other advantages: (a) Compared with conventional sensing, multi-layer sensing is often more efficient, in terms of both performance and system structure simplicity. (b) At any given time, information about an external world object may only reveal finite aspects, that may make the compound sensing impractical. (c) Complex information usually consists of high dimensional variables, processing too many of them simultaneously will result in a significant increase of time and cost ${ }^{[12],[13]}$.

\section{Measurement of temperature, humidity, and brightness by way of multi-layer sensing}

2.1 Principle of the sensor A new multifunctional sensor incorporating $\mathrm{Fe}_{3} \mathrm{O}_{4}$ with $\mathrm{CdS}$ for the measurement of humidity, temperature and brightness has been developed, which structure is shown in Fig.5. The electrical characteristics of $\mathrm{CdS}$ and $\mathrm{Fe}_{3} \mathrm{O}_{4}$ had been found to be sensitive to brightness and humidity, respectively, and also to temperature as well as other semi-conductive materials ${ }^{[9]}$.

Consider the equivalent circuit of the sensor shown in
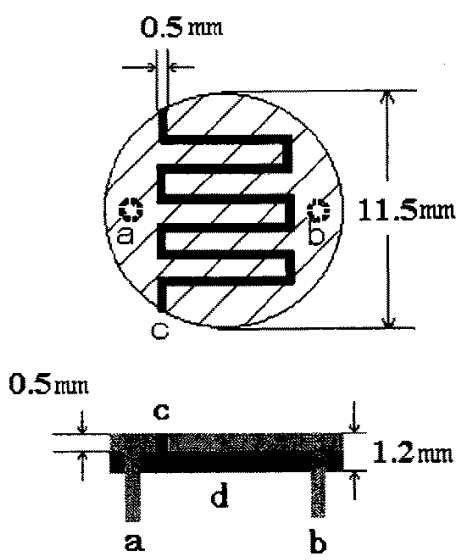

a,b-copper electrodes

c- sensing materials, mixed with $\mathrm{Fe}_{3} \mathrm{O}_{4}$ and

CdS

d- insulated substrate

Fig. 5 Structure of the sensor.

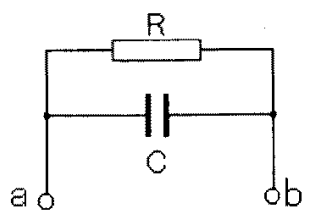

Fig.6 Equivalent circuit of the sensor.

Fig.6, there is a two-dimensional pair as the sensor outputs specified by resistance $R$ and capacitance $C$ :

$$
\begin{aligned}
& R=f\left(v_{H}, v_{T}, v_{B}\right) \\
& C=g\left(v_{H}, v_{T}, v_{B}\right)
\end{aligned}
$$

Where, $v_{i:}, v_{r}, v_{B}$ are the sensor input values of humidity, temperature and brightness, respectively; Like equation (1), $f$ and $g$ are general forward functions denoting the relationships between the inputs and outputs of the sensor. However, there is one more quantity that is under measured in equation (2) than in equation (1).

Resistance effect of the sensor comes from energy transition of the particles inside the sensitive materials. When the particles get stimulation from ray radiation or the affinity from water molecules, and if the energy they each received is more enough, they could be resolved as electrons (jumping to transmit zone) and holes (jumping to valence zone). Thus, the electrical conductivity of the 
sensor varies with the environment quantities. Capacitance effect generates from dielectric characteristic variation of the sensing device caused by the quantities.

2.2 Experimental setup of the sensor All experiments are carried out within a lounge, in which both temperature and humidity can be appointed. The experimental setup of the sensor is shown in Fig.7.

Where inside the lounge, temperature value could be controlled from $-10^{\circ} \mathrm{C}$ to $60^{\circ} \mathrm{C}$ (with the resolution being $0.1^{\circ} \mathrm{C}$ ), and humidity could be adjusted from $20 \% \mathrm{Rh}$ to $100 \% \mathrm{Rh}$ (the resolution is $1 \% \mathrm{Rh}$ ). Luminary control unit is attached to make the brightness varies from 0 to $1500 \mathrm{Lx}$ (only rough variation could be given). To get the precise information about the quantities that affect the sensor, the calibration meters are also set up to measure the three variables, respectively. The resistance and capacitance values (outputs of the sensor) were measured by a LCR meter, from which the signal is interfaced with the computer for data processing

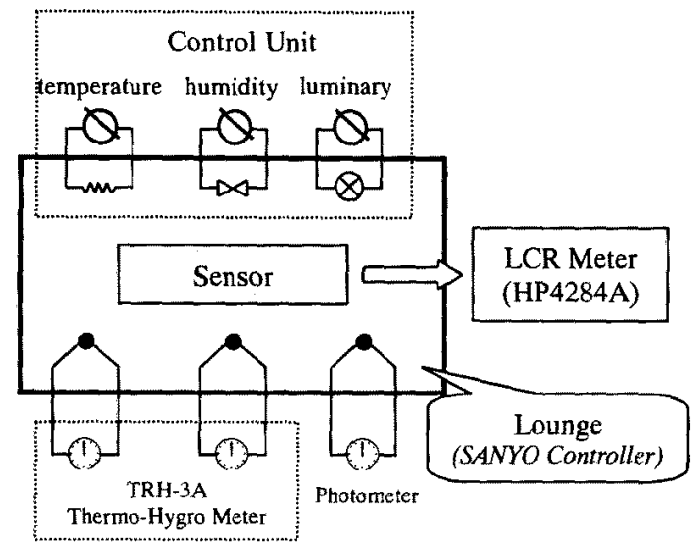

Fig.7 Experimental setup of the sensor

2.3 Selection of the measurement equations It is impossible to estimate the whole three measurand values from (2) only, because its inverse function is obviously ill posed. (For ill posed measurement equations, the evaluation of few variables could be available at some special occasions $^{[6]}$ ) By adjusting the working frequency of the $L C R$ meter used in the measurement, we may get the output results in two groups with the different frequency values:

$$
\begin{aligned}
& \left\{\begin{array}{l}
R_{1}=f\left(v_{H}, v_{T}, v_{B}, \omega_{1}\right)=\left.f_{1}\left(v_{H}, v_{T}, v_{B}\right)\right|_{\omega-\omega_{1}} \\
C_{1}=g\left(v_{H}, v_{T}, v_{B}, \omega_{1}\right)=\left.g_{1}\left(v_{H}, v_{T}, v_{B}\right)\right|_{\omega-\omega_{1}}
\end{array}\right. \\
& \left\{\begin{array}{l}
R_{2}=f\left(v_{H}, v_{T}, v_{B}, \omega_{2}\right)=\left.f_{2}\left(v_{H}, v_{T}, v_{B}\right)\right|_{\omega-\omega_{2}} \\
C_{2}=g\left(v_{H}, v_{T}, v_{B}, \omega_{2}\right)=\left.g_{2}\left(v_{H}, v_{T}, v_{B}\right)\right|_{\omega-\omega_{2}}
\end{array}\right.
\end{aligned}
$$

Then considering the final impedance $Z_{a b}$ in Fig.6, we have

$$
Z_{a b}=\frac{R}{1+\omega^{2} C^{2} R^{2}}-j \frac{\omega C R^{2}}{1+\omega^{2} C^{2} R^{2}}
$$

The Equation (5) implies that the real part of the impedance (resistance) is much inert to frequency $\omega$, compared with the imaginary part (capacitance). Relevantly we can select the measurement equations as below,

$$
\left\{\begin{array}{l}
R=\left.f\left(v_{H}, v_{T}, v_{B}\right)\right|_{\omega-\omega_{1} \text { or } \omega_{2}} \\
C_{1}=\left.g_{1}\left(v_{H}, v_{T}, v_{B}\right)\right|_{\omega-\omega_{1}} \\
C_{2}=\left.g_{2}\left(v_{H}, v_{T}, v_{B}\right)\right|_{\omega-\omega_{2}}
\end{array}\right.
$$

If $g_{1}$ and $g_{2}$ are independent, or $g_{1} / g_{2} \neq \omega_{1} / \omega_{2}$, then (6) can be theoretically used for the evaluation of the quantities being measured, that is, the values of $v_{H}, v_{T}$ and $v_{B}$ may be estimated from the actual measurement results of $R, C_{1}$ and $C_{2}$, as follows:

$$
\left\{\begin{array}{l}
v_{H}=\Gamma_{1}\left(R, C_{1}, C_{2}\right) \\
v_{T}=\Gamma_{2}\left(R, C_{1}, C_{2}\right) \\
v_{B}=\Gamma_{3}\left(R, C_{1}, C_{2}\right)
\end{array}\right.
$$

Where, $\Gamma_{1}, \Gamma_{2}, \Gamma_{3}$ are inverse group functions of (7).

Then we propose to lower the rank of (6) according to the gyroscopic effects of the sensor, and try to ascertain the variables $\left(v_{H}, v_{T}, v_{B}\right)$ asynchronously to avoid reconstructing the quantities in high dimension space.

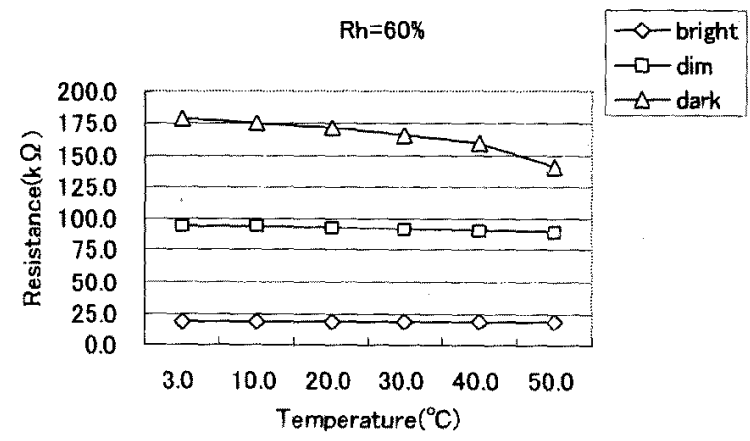

Fig.8 The relationships between resistance and temperature while brightness changes

It has been proved that the change of brightness or humidity can only stimulate the amount of free ions or holes on the surface of sensing material, while temperature also impacts the inside. Experiment data in Fig. 8 shows that the change of the resistance value of the sensor for temperature also varies with the different brightness conditions. If the dark surroundiny condition serves (among the three quantities, only brightness can be modulated to satisfy the measurement request without annoying the three quantities themselves), temperature value can influence the resistance of the sensor. Thus, two kinds of resistance are to be totally examined instead of only one:

$$
\begin{aligned}
& R_{0}=f\left(v_{H}, v_{T}, v_{B}^{*}\right) \\
& R=f\left(v_{t H}, v_{T}, v_{B}\right)
\end{aligned}
$$

$v_{B}^{*}$, denoting the value of darkness, can be realized by periodically assessing the sensor to a closed and absolute dark lounge during the measurement. By using the measurement data of $R_{0}$ and $C_{1}$ (or $C_{2}$ ), temperature and 


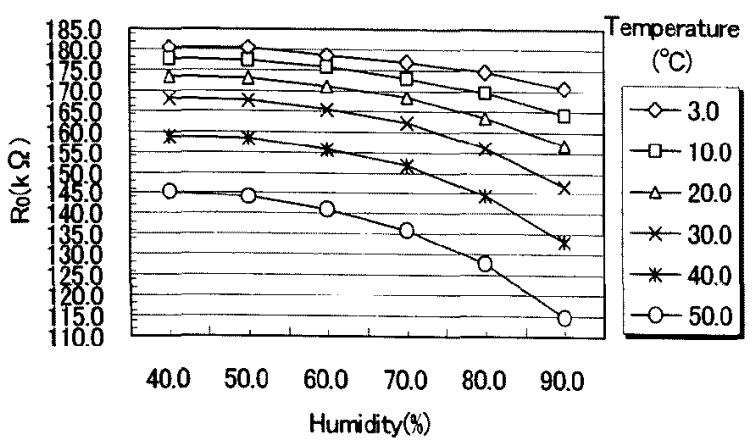

Fig.9 The relationships between resistance and humidity while temperature changes.

humidity values are to be reconstructed, and finally brightness value can be evaluated on the measurement of $R$ in equation (8).

\section{Experimental Results and Discussions.}

3.1 Reconstruction of quantities The experiment of humidity effects to resistance $\left(R_{0}\right)$ are shown in Fig. 9.

In fact, the value of the measured capacitance can be deduced from the imaginary part of (5):

$$
C_{X}=\frac{1}{\omega^{2} C R^{2}}+C
$$

When the instrument frequencies are set to be $\omega_{1}$ and $\omega_{2}\left(\omega_{2}>\omega_{1}\right)$, respectively, the capacitances $C_{1}$ and $C_{2}$ in (6) of the sensor are measured as

$$
\begin{aligned}
& C_{1} \equiv C_{X 1}=\frac{1}{\omega_{1}^{2} C R^{2}}+C \\
& C_{2} \equiv C_{X 2}=\frac{1}{\omega_{2}^{2} C R^{2}}+C
\end{aligned}
$$

To estimate the value of humidity, instead of using the function $C_{1}$ (or $\mathrm{C}_{2}$ ), $C$ is selected in the reconstruction calculation, so as to improve the measurement dynamical characteristics. Compared with the resistance being influenced by both humidity and temperature, humidity variation affects the surface of sensing material more quickly because of the change of its dielectric nature. The

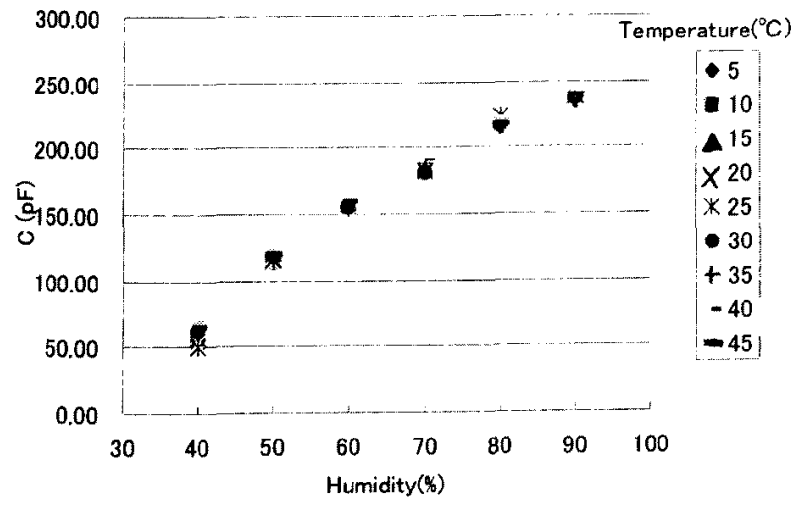

Fig.10 Relationships between humidity and $C$ value of $C$ can be calculated by (10) and (11):

$$
C=C_{2}-\frac{1}{\left(\omega_{2} / \omega_{1}\right)^{2}-1}\left(C_{1}-C_{2}\right)
$$

Based on (12) and with the dark condition of surrounding serving, the result of the humidity measurement is shown as Fig.10, on which the impact of temperature can be seen to be effectively restrained. Thus, humidity value can be evaluated principally based on the capacitance value with the measurement of resistance being considered as the calibration term.

In accordance with the preceding discussion, three kinds of outputs are measured in the process:

$$
\begin{aligned}
& C=C_{2}-\frac{1}{\left(\omega_{2} / \omega_{1}\right)^{2}-1}\left(C_{1}-C_{2}\right)=g\left(v_{H}, v_{T}, v_{B}^{*}\right) \\
& R_{0}=f\left(v_{H}, v_{T}, v_{B}^{*}\right) \\
& R=f\left(v_{H}, v_{T}, v_{B}\right)
\end{aligned}
$$

Where, $R_{0}$ is the resistance in dark condition, and $R$ is the resistance when sensor is exposed in the radiance of environment. To get the exact values of humidity, temperature and brightness, practical measures are carried out in the following three layers.

(1). At first layer, placing the sensor in dark environment, measuring its capacitance and resistance, then evaluating the humidity value based on the data shown in Fig.11. Least mean squares (LMS) algorithm is used here to approach the converse solutions of the nonlinear functions. The reconstruction is based on the specimen data of $C$ and $R_{0}$, which are specified by the partial equations of (13):

$$
\left\{\begin{array}{l}
C=C_{2}-\frac{1}{\left(\omega_{2} / \omega_{1}\right)^{2}-1}\left(C_{1}-C_{2}\right)=g\left(v_{H}, v_{T}, v_{B}^{*}\right) ; \\
R_{0}=f\left(v_{H}, v_{T}, v_{B}^{*}\right) .
\end{array}\right.
$$

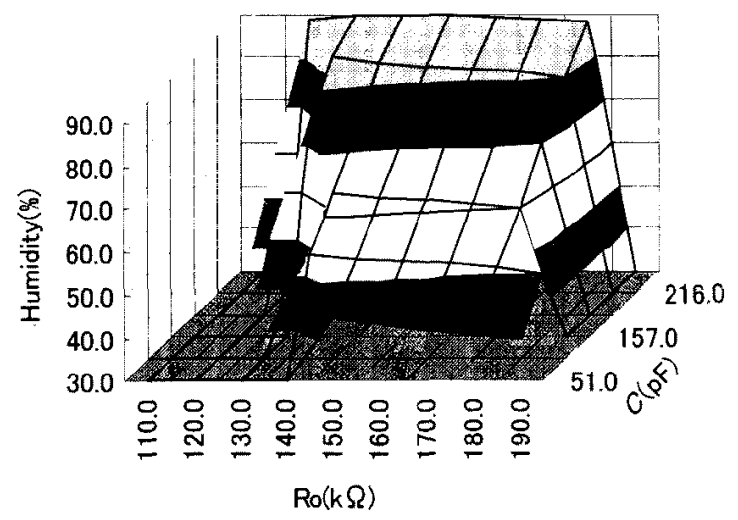

Fig. 11 Humidity value reconstruction

(2). At the second layer with the humidity value known in the same condition above, the temperature value based on the data shown in Fig.12 is estimated, which is reconstructed on the equation, $R_{0}=f\left(v_{H}, v_{T}, v_{B}^{*}\right)$.

Temperature can also be reconstructed on the measurement of $C$ and $R_{0}$, as well as in the first layer. The 
reasons of using known humidity value instead of $R_{0}$ and $C$ here to evaluate temperature might be:

a. Convenient for the computation of reconstruction. As we now have described in the paper, Least Mean Square (LMS) method was used for the estimation of humidity by the interpolation of $1^{\text {st }}$ order polynomial segments to connect the particular measured samples. The method works in $3 \mathrm{D}$ space, and could be considered as twodimensional signal processing. However, for estimation of temperature, algorithm could be simplified since the processing now can be performed as one-dimensional processing.

Consider a nonlinear equation as $y=f\left(x_{1}, x_{2}\right)$. If $x_{1}$ is already known, then $y$ is actually a mono function of $x_{2}$, that is

$$
y=f\left(x_{1}, x_{2}\right)=\left.g_{1}\left(x_{2}\right)\right|_{x_{1}-k_{1}}=\left.g_{2}\left(x_{2}\right)\right|_{x_{1}-k_{2}}=\ldots \ldots
$$

Thusly the inverse function of $g_{1}, g_{2}, \ldots$ could simply be computed by way of mono mean square regression.

b. The curved surface of the solution is relatively smooth compared using $C$ and $R_{0}$. In this case, the length of stages in the computation of LMS method could be bigger to approximate the nonlinear functions under same accuracy request.

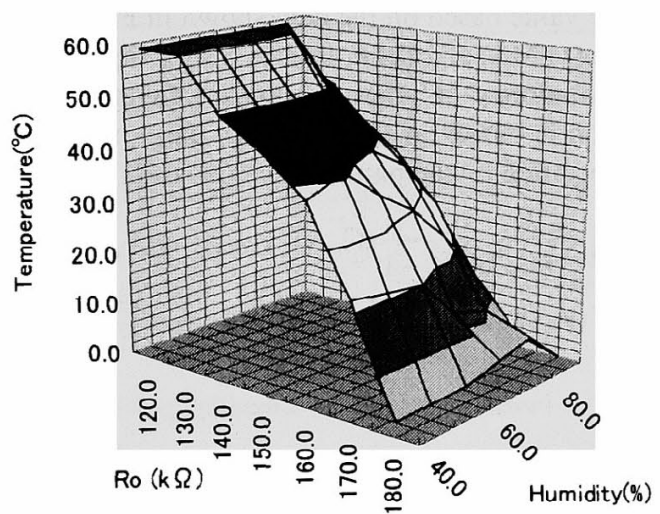

Fig.12 Temperature value reconstruction

(3). Finally at the third layer, exposing the sensor to the real surroundings (let the rays come in), and with the influences on resistance by humidity and temperature having been eliminated, the brightness value is assessed. Example data is shown in Fig.13 in the case that

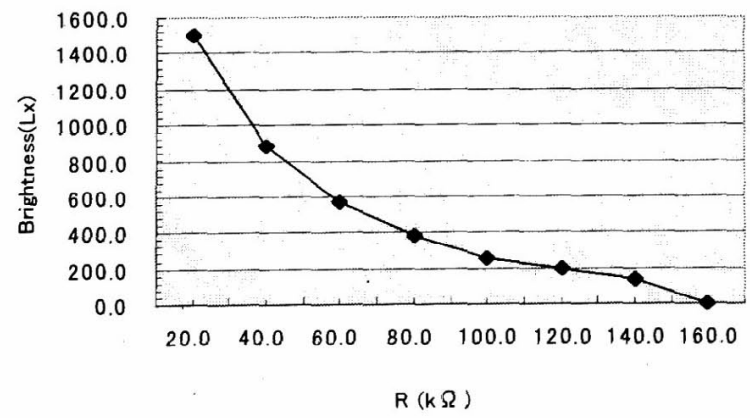

Fig.13 Brightness value reconstruction temperature and humidity are $20^{\circ} \mathrm{C}$ and $65 \% \mathrm{Rh}$, respectively.

Example measurement results for the controlled small lounge are given in Tab. 1. Where, "Appointed value" is appointed by the control unit of the lunge and is calibrated by several meters. "Measured value" generated from the proposed sensor.

3.2 Uncertainty of the measurement The difference between "Appointed value" and "Measured value" denoted the measurement uncertainty of the proposed sensor.

For the case of humidity evaluation in this research, it can be considered that the nonlinear two-dimensional functions $y_{1}=f\left(x_{1}, x_{2}\right)$ and $y_{2}=g\left(x_{1}, x_{2}\right)$ were substituted by multi-1 ${ }^{\text {st }}$ polynomial ones (It means to separate both $y_{1}$ and $y_{2}$ with smaller regions). For example, the variation range of $y_{1}$ is considered as $\left[y_{11}, y_{12}\right],\left[y_{12}, y_{13}\right], \ldots \ldots,\left[y_{1(\mathrm{~N}-1)}\right.$, $y_{\mathrm{N}}$.

And within each smaller region, we have

$$
y_{1}=a x_{1}+b x_{2}+1 \text {. }
$$

In the same way for $y_{2}$, we have

$$
y_{2}=c x_{1}+d x_{2}+1 \text {. }
$$

The parameters ( $a, b, c$ and $d$ ) of (16) and (17) are estimated by applying LMS algorithm on the sensing data within each smaller regions. Thus, the values of $x_{1}$ and $x_{2}$ could be calculated out by the solutions of (16) and (17) as below

$$
\begin{aligned}
& \hat{x}_{1}=\frac{d y_{1}-b y_{2}+b-d}{a d-b c} \\
& \hat{x}_{2}=\frac{c y_{1}-a y_{2}+a-c}{b c-a d} .
\end{aligned}
$$

Either $\hat{x}_{1}$ or $\hat{x}_{2}$ is actually a square plane in threedimensional space (the coordinates are on the independent variables $y_{1}$ and $y_{2}$, respectively), The whole regression solution plane is composed of such smaller inclined ones, of which the number depends on the amount of the divided regions of both $y_{1}$ and $y_{2}$.

Define the uncertainty error of $x_{1}$ as $d \hat{x}_{1} / \hat{x}_{1}$, then from (18), we have

$$
\begin{gathered}
\Delta \hat{x}_{1} / \hat{x}_{1} \approx \\
\frac{\left(d \cdot \Delta y_{1}+\Delta d \cdot y_{1}\right)+\left(b \cdot \Delta y_{2}+\Delta b \cdot y_{2}\right)+\Delta b-\Delta d}{d y_{1}-b y_{2}+b-d}+ \\
\frac{(a \cdot \Delta d+\Delta a \cdot d)-(b \cdot \Delta c+\Delta b \cdot c)}{b c-a d}
\end{gathered}
$$

Where, $\Delta y_{1}, \Delta y_{2}-$ system error;

$$
\Delta a \sim \Delta d \text { - LMS algorithm error. }
$$

System error resulted from the accuracy of instruments, calibration error of the meters that were used for monitoring the measurand, the stability of the sensor itself and the controlling performance of the lunge. LMS error existed in the reconstruction process where we approximated groups of linear equations to the nonlinear functions of the sensor. Electrical-magnetic noise may also influence the accuracy of the sensor.

System error is to be restrained by improving the experiment conditions and the technological manufacture 
Multi-layered Sensing Approach for Environment Perception

Tab.1 Measurement results

\begin{tabular}{|c|c|c|c|c|c|c|}
\hline & \multicolumn{3}{|c|}{ Appointed value } & \multicolumn{3}{c|}{ Measured value } \\
\cline { 2 - 7 } & Humidity $(\%)$ & Temperature $\left({ }^{\circ} \mathrm{C}\right)$ & Brightness $(\mathrm{Lx})$ & Humidity $(\%)$ & Temperature $\left({ }^{\circ} \mathrm{C}\right)$ & Brightness $(\mathrm{Lx})$ \\
\hline 1 & 40.0 & 5.0 & 0 & 47.6 & 12.8 & 50 \\
\hline 2 & 40.0 & 5.0 & 1350 & 45.2 & 7.8 & 1300 \\
\hline 3 & 40.0 & 45.0 & 1450 & 50.8 & 37.9 & 1400 \\
\hline 4 & 90.0 & 25.0 & 350 & 85.4 & 21.6 & 450 \\
\hline 5 & 65.0 & 25.0 & 1450 & 59.3 & 22.4 & 1400 \\
\hline 6 & 90.0 & 40.0 & 0 & 92.2 & 43.5 & 100 \\
\hline 7 & 90.0 & 40.0 & 350 & 95.7 & 46.4 & 450 \\
\hline 8 & 65.0 & 25.0 & 1150 & 70.6 & 29.8 & 1200 \\
\hline
\end{tabular}

levels of the sensor; Introducing of other interpolation functions (e.g. spline function) is expected to reduce the LMS algorithm error.

3.3 Sensitivity of the sensor The sensitivity of the sensor is mainly decided by the values of local (partial) derivative of the forward measurement functions. For example, considering the humidity measurement, from (14) we have

$$
\left\{\begin{array}{l}
\frac{\partial R_{0}}{\partial v_{H}}=\frac{\partial f\left(v_{H}, v_{T}, v_{B}^{*}\right)}{\partial v_{H}} \\
\frac{\partial C}{\partial v_{H}}=\frac{\partial g\left(v_{H}, v_{T}, v_{B}^{*}\right)}{\partial v_{H}}
\end{array}\right.
$$

The values of the two derivatives obviously had determined the gradient of the camber shown of Fig.11, on which the sensitivity on humidity measurement of the sensor is given. Generally, the bigger the derivatives are, the higher the sensitivity is. Although the measurement results were finally from the reconstruction computations, the (LMS) algorithm could not change the characteristics of sensitivity in essence.

Since the measurement equations are non-linear ones, the sensitivity of the sensor is therefore not constant within the whole measurement range. Furthermore, the sensitivity of one quantity was also influenced by the value of other quantities. For example, when humidity and temperature are $65 \% R h$ and $20^{\circ} \mathrm{C}$, respectively, the sensitivity of brightness sensing changed from $2.5(L x / k \Omega)$ to 32.5 $(L x / k \Omega)$.

To improve the sensitivity of the sensor, modification on the technological design of the sensor is needed.

\section{Conclusions}

In this approach, we use only one multi-functional sensor to measure three environment variables in a step by step way, of which the technique deserves the tactics of the multi-layer sensing.

Our proposed multi-layer sensing technique provides a possible strategy for the solution to measurement equations for multi-functional sensor, especially when the sensor processes more than three quantities that cannot be reconstructed in parallel by the input/outputs data of the sensor. The variables being measured could probably be classified into different layers or groups considering their instinctive nature to the output of the sensor, for example, the non-linear sensitivity, frequency characteristics, and so on. Thus, the multivariate equations were lowered to avoid computing the data in high dimension space. However, both the sensing device and the reconstruction algorithm need modification to reduce the measurement uncertainty and enlarge the sensitivity. Attempts at modifying the sensor will be made in the follow-up research.

Furthermore, the universal solutions to measurement equations of nonlinear form are still far from realization, more fruitful approaches in this field are under expectation.

On other hand, the processing of high-level information that is related to many conventional physical or other kinds of quantities has rapidly evolved recently ${ }^{[1],[2],[14]}$. Environment "comfort" can be considered as such a type of information that comprises temperature, humidity and other variables. High-level information is of great importance to intelligent systems (like robotics), in which it is needed to extract and fuse data from multi-sensor systems to acquire more accurate information about the external environment. Multi-layer information fusion that sequentially aggregates information is proposed here. Unlike traditional fusion methods, multi-layer fusion might probably be possessed of the advantage of high efficiency and dependency. Further explorations now still wait for making dependant on [15].

\section{Acknowledgments}

The authors would like to thank Dr. J. Yuji, Dr. Y.G. Xiao, Mr. W. Yoshida, and Dr. A. Kimoto for their valuable advice and help. The supports of other members in SHIDA Group (Saga University) to this work are also greatly appreciated.

(Manuscript received Aug.9, 1999, revised Dec.3, 1999.)

\section{References}

[1] G.Mauris, E. Benoit, L. Foulloy, The aggregation of complementary information via fuzzy sensors, Measurement, vol.17, No.4, pp.235-249, 1996.

[2] Kai-tai Song, Wen-hui Tang, Environment perception for a mobile robot using double ultrasonic sensors and a CCD camera, IEEE Trans. On Industrial Electronics, vol.43, No.3, June, 1996, pp.372-379.

[3] K. Shida and D. Li, A mono-structural Thin Plate touch Sensor with Four Sensing Functions for Discriminating of 
the Material Properties, Proc. of the IEEE IMTC, pp 152, 1995.

[4] K. Shida and J. Yuji, Discrimination of Material Property by Pressure-Conducting Rubber Sheet Sensor with MultiSensing Function, Proceedings. of the IEEE Int Symposium on Industrial Electronics, pp. 54, 1996.

[5] Abu Bakar Md. Ismail, and Katsunori Shida, Non-contact multi-sensing technique for the precise measurement of concentration of electrolytic solution, Sensors and Actuators, vol. 69/2, August, 1998.

[6] Tomasz Szafranski, et al., Accuracy of measurand, IEEE Instrumentation and Measurement Technology Conference, St. Paul, USA, May 18-21, 1998, pp.32-35.

[7] Akira Kobayashi, Measurement equations, Journal of the society of instrument and control engineers, Vol.27, No.5, 1988, pp.383-388.

[8] Susan S. Young, Peter D. Scott, Object recognition using multilayer Hopfield neural network, IEEE Trans. on Image Processing, Vol.6, No.3, March 1997, pp.357-372.

[9] Xianda Cheng, Modern signal processing, Tsing Hua Publishing House, May, 1995.

[10] Shinji OHYAMA, and et al., Zooming functional position measurement in enclosed signal field as progressive learning measurement system, Technical Digest of The 16th Sensor Symposium, 1998, pp.105-110.

[11] Toshiharu Mukai, Masatoshi Ishikawa, An active sensing method using estimated errors for multisensor fusion systems, IEEE Trans. On Industrial Electronics, vol.43, no.3, June 1996, pp.380-385.

[12] Tony Gustafsson, Instrumental variable subspace tracking using projection approximation, IEEE Trans. On Signal processing, vol.46, no.3, March 1998, pp.669-681.

[13] Steven G. Goodridge, and et al., Multilayered fuzzy behavior fusion for real-time reactive control of systems with multiple sensors, IEEE Trans. On Industrial Electronics, vol. 43, no. 3, June 1996, pp.387-394.

[14] Peter Wide, and et al., The human-based multi-sensor fusion method for artificial nose and tongue sensor data, IEEE Instrumentation and Measurement Technology Conference, St. Paul, Minnesota, USA, May 18-21,1998, pp.531-536.

[15] Jinwei Sun and Katsunori Shida, Fuzzy algorithms of information fusion for multi-layer sensing, 電気学会研 究会資料, IM-99-48.

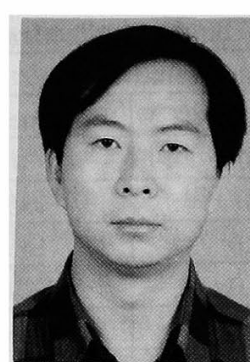

Jinwei Sun (non-Member) received his M.S. degree in electrical engineering from Harbin Institute of Technolngy (HIT), P.R. China in 1990. Since 1990 he has been a lecturer in HIT. From 1997 up till now, he is a Ph.D. student in Dept. of E.E., Faculty of Sci. and Eng., Saga University, Japan. His main research interests include sensor technology, sensor fusion, instrumentation, industrial control and signal processing.

Katsunori SHIDA (Member) obtained his M.Sc. and Ph.D degrees in Electronic Engineering from Tohoku University,

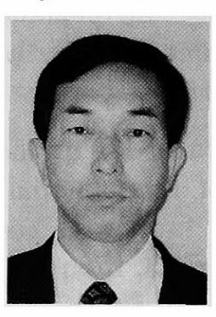

Sendai, Japan in 1970 and 1981, respectively. He joined the Electro-technical Lab (ETL), Ministry of International Trade and Industry, Japan in 1970. Since 1990 he has been a professor of Saga University and engaged in the research on intelligent precision electric measurement and instrumentation. $\mathrm{He}$ is an honorable member at many academic and professional societies in Japan and abroad. 